\title{
Scanning tunneling microscopy of the phosphatidylcholine bilayers
} \author{
Zhengbo Cheng, and Chunli Bai \\ Institute of Chemistry, Academia Sinica, Beijing, China \\ Yaxian Su, Sanduo $\mathrm{Xu}$, and Kechun Lin \\ Department of Cell Biology, Beijing Medical University, Beijing, China \\ John D. Baldeschwieler \\ California Institute of Technology, Pasadena, California 91125
}

Changhong Luo, Chuanfeng Zhu, Like Ruan, Guizhen Huang, Changchun Dai,

(Received 10 July 1989; accepted 23 August 1989)

\begin{abstract}
The bare surface of the egg-PC bilayers after being dried at room temperature was observed by the STM and images with angstrom resolution were obtained. Results indicate that the lipid heads form islands of an average of $210 \AA$ in diameter and $40 \AA$ in separation on the bilayers and that each island contains about 170 lipid heads of $7.5 \AA$ in diameter. The size of the lipid heads is correspondent with that calculated from the standard bond distances and the bond angles. The ordered distribution of the lipid heads in some areas may correspond to the ordered arrangement of the lipid heads on the egg-PC bilayers before being dried. The STM results also show that there exists the ripple phase in the egg-PC bilayers below its phase transition temperature.
\end{abstract}

\section{INTRODUCTION}

The study of biomembrane is very important as we know. The cell is a basic working unit for living and the biomembrane system in the cell is a place for performing the functions of cells such as material transport, energy transformation, and information transfer, etc. The basic compositions of the biomembrane are lipids and protein molecules. The plasma membrane of a cell is made up of many kinds of lipids, which compose the bilayers membrane as a basic skeleton, and then various kinds of proteins are attached or sinked in the lipid bilayers, so as to form the so-called liquid mosaic membrane structure. The composition and the structure of the natural biomembrane is very complex, for the sake of convenience, we used the artificial lipid bilayers membrane at first to study the structure of the membrane.

The scanning electron microscope (SEM) and the transmission electron microscope (TEM) were used as tools to study the structure of biomembrane and lipid bilayers membrane, but their resolutions were limited for analyzing the structure variations of biomembrane. The scanning tunneling microscope (STM) has been proven to be an excellent tool in detecting the structural and electronic properties on the atomic scale on metal, semiconductor, and superconductor surfaces. The fact that STM can be performed not only in vacuum, but also in ambient conditions, and even in isolating liquids, makes it possible to apply the superior resolving power of the STM to biomaterials. A limited number of biological macromolecules have been imaged with the STM, including DNA in vacuum ${ }^{1}$ and in water, ${ }^{2}$ metal-shadowed DNA, ${ }^{3}$ a globular protein, ${ }^{4}$ bacteriophage $\Phi-29,,^{5}$ porin vesicles, ${ }^{6}$ and the ripple phase of dimyristoylphosphatidylcholine (DMPC) bilayers. ${ }^{7}$

The difficulties in imaging biomaterials with STM is that most biological materials are liquid, with rough surfaces and poor conductivity. So the samples' deformation, Brownian motion, and other thermal fluctuations are large. Though glass, gold or carbon cover may lessen their Brownian motion and thermal fluctuations, the results are somewhat rough and the resolution is limited. So the bare surface of the egg-PC bilayers was used in our experiments. Furthermore, water plays an important role in the characteristics of the biomaterials. What would its structure be if the egg-PC bilayers was dried before being observed? To reveal it, a dried sample was used. In our experiments, we reveal the surface structure of the dried egg-PC bilayers with angstrom resolution.

\section{SAMPLE PREPARATION AND EXPERIMENTAL}

The sample was prepared by dissolving $0.5 \mathrm{mg}$ of egg-PC in $0.2 \mathrm{ml}$ of trichloromethane, rotate evaporating in vacuum for $1 \mathrm{~h}$, hydrating with $0.2 \mathrm{ml}$ of metal-free water, sonicating in a water bath five times ( $1 \mathrm{~min} /$ time), then dropping a little of the such treated sample on small stainless steel plates. It then air dried for $3 \mathrm{~h}$ naturally in glass container, fixing the stainless steel plate with egg-PC bilayers on it on the sample table of the STM and imaging its bare surface at room temperature.

The experiment was performed with CSTM9000 (Institute of Chemistry, Academia Sinica, Beijing, Peoples Republic of China). The bias voltage was $80-90 \mathrm{mV}$, with the sample positive with respect to the tip. The tunneling current was $1 \mathrm{nA}$. Images were obtained in constant current mode at room temperature and ambient pressure. The transverse calibration of the microscope was established by imaging the well-known surface of graphite. Tip movement and images recording were all performed by a computer monitor. The tip used was gotten by etching a $0.5 \mathrm{~mm}$ diameter tungsten tip in a $2 \mathrm{~N} \mathrm{NaOH}$ solution at the voltage of $13 \mathrm{~V}$ dc.

\section{RESULTS AND DISCUSSION}

Figure 1 is the STM image of the surface of the egg-PC bilayers taken with different scanning ranges. Figure 1(a) was taken from a large area. According to the tunneling effect principle, STM results combine both structural and electronic informations, where the electronic densities are large 

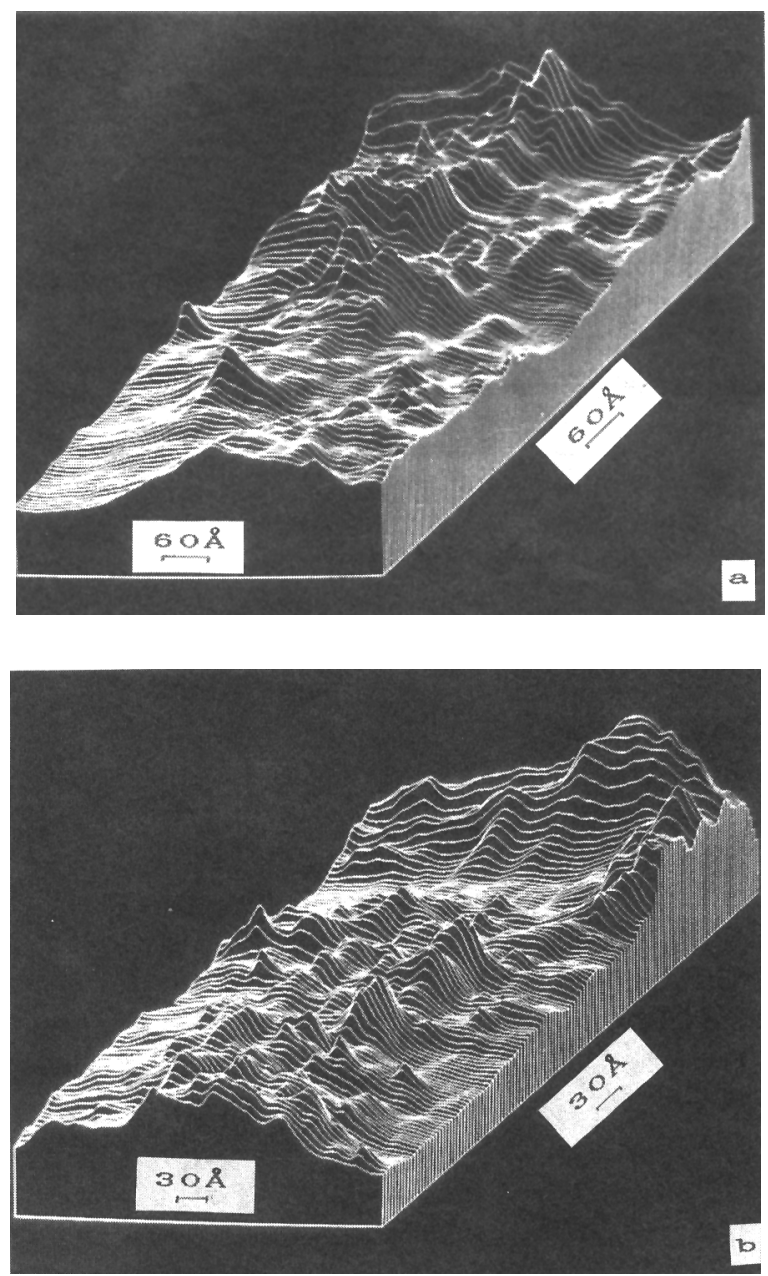

FIG. 1. STM images of the surface of the dried egg-PC bilayers taken with different scanning ranges. (a) Overview. Islands of an average of $210 \AA$ in diameter and $40 \AA$ in separation are apparently seen. The islands were formed by the contraction of the egg-PC bilayers when being dried. (b) The magnified image of one island. Each island contains $\sim 170$ lipid heads. Each lipid head is an average of $7.5 \AA$ in diameter and the average distance between the heads is $\sim 16 \AA$. Although these results come from the dried sample, they correspond with those calculated from the standard bond distances and the bond angles of the lipid's formula. This may be evidence that the drying process does not change the sample's internal electronic structure.

at peaks in the image compared with those at plane areas. In the case of the egg-PC bilayers, peaks in the image contain the space scale of arrangement of lipid heads. In this figure, we can see many islands on the film. The size of the islands and the separation between islands were measured by using the topological method. That is, by drawing three vertical lines across the figure, measuring the average cut sizes of the islands and the separations on each line, and taking the average of the cut sizes on the three vertical lines. Islands were measured $\sim 210 \AA$ in diameter and $40 \AA$ in separation by using this topological method. It is not surprising for the egg-PC bilayers membrane being composed of lipid heads. When the sample was dried; the PC bilayers contracted and so the lipid heads on it formed islands. Figure 1(b) was taken from a smaller area. It shows the more fine structure of the egg-PC bilayers. From the image, by using the topologi- cal method described above, we can find that each lipid head is an average of $7.5 \AA$ in diameter and the average distance between the heads is $\sim 16 \AA$. From Fig. 1(a), the island is $210 \AA$ in diameter. That is, each island contains about 170 lipid heads of $\sim 16 \AA$ in separation. Although these results come from the dried sample, they correspond with those calculated from the standard bond distances and the bond angles of the lipid's formula:<smiles>CCC(=O)OCC(COP(=O)([O-])OCC[N+](C)(C)C)OC(=O)CC</smiles>

the lipid tail the lipid head.

This may be the evidence that the drying process does not change the sample's internal electronic structure.

According to Fig. 1, we can conclude that lipid heads form islands on the egg-PC bilayers during the drying process. Each island is $210 \AA$ in diameter and $40 \AA$ in separation. Each island contains 170 lipid heads. Each lipid head is $7.5 \AA$ in diameter and the average distance between the heads is an average of $16 \AA$. The correspondency of the lipid's size and separation with those calculated from the lipid's formula may be one of the evidences that the drying process does not change the sample's internal electronic structure. The results themselves are not very important but they are especially important in the field of STM for that, as we have indicated in the introduction, for facing the biomaterials' poor conductivity, deformation, Brownian motion,

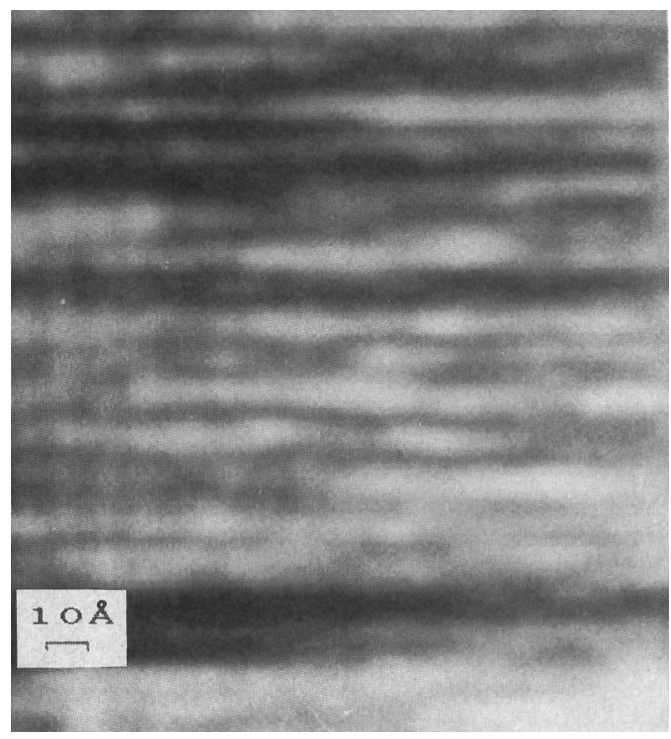

FIG. 2. The ordered distribution of the lipid heads in some areas on the eggPC bilayers (as is shown in the left area). This may imply that the lipid heads were in ordered arrangement before the sample was dried. The left area in the image showed one of those areas in which the ordered distribution were reserved after the sample was dried. 


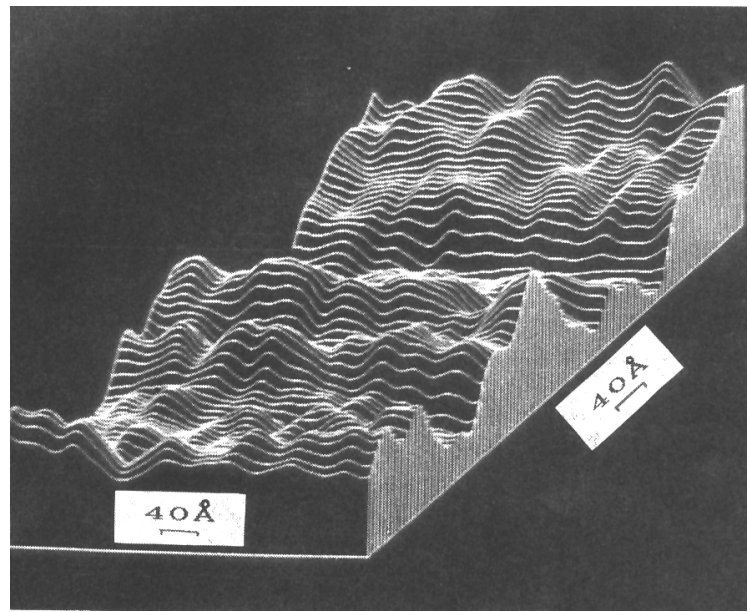

FIG. 3. The ripple phase of $128 \AA$ in period observed in the egg-PC bilayers below its phase transition temperature. The ripple phase is believed to be an alternative way of satisfying the head-tail area incompatibility.

and other thermal fluctuations. Gold, carbon, or glass covers and copies were often used in the STM observation. In this paper, we imaged the bare surface of the biomaterials directly and obtained angstrom resolution. The fact that STM can be applied directly to the poor conductivity, soft surface of biomaterial may imply that the tunneling effect may have an effect even in more expanded fields than we have expected.

Figure 2 is the STM image taken from another area. From this image, we can find that the distribution of the lipid heads is ordered in some areas, as is shown in the left area in the image. The ordered distribution of the heads in some areas may imply that the distribution of the lipid heads in the bilayers was ordered before it was dried. What we have observed is one of those areas in which the ordered distribution was reserved.

Figure 3 is the ripples observed in some areas. Each ripple is $128 \AA$ in period. Joseph et al. ${ }^{7}$ have observed the ripple phase of $120-130 \AA$ of the DMPC bilayers below its main phase transition temperature $24^{\circ} \mathrm{C}$ and above $14^{\circ} \mathrm{C}$. The DTA result shows that the phase transition temperature of the egg-PC bilayers is $\sim 22-25^{\circ} \mathrm{C}$. The STM image was tak- en at room temperature of $\sim 22^{\circ} \mathrm{C}$. The STM result shows that there also exists the ripple phase in the egg-PC bilayers below its phase transition temperature. The ripple phase is believed to be an alternative way of satisfying the head-tail area incompatibility.

Although these results contributed come from the studies on the simple artificial liposomes, they are essential to the studies of the relationship between the changes on the molecule arrangement of the biomaterials under various factors and the action for living.

\section{CONCLUSIONS}

(1) The lipid heads form islands of $210 \AA$ in diameter and $40 \AA$ in separation during drying process. Each island contains $\sim 170$ lipid heads of $7.5 \AA$ in diameter. The correspondence of the lipid's size and separation with those calculated from the lipid's formula may be one of the evidences that the drying process does not change the sample's internal electronic structure.

(2) The distribution of the lipid heads on the egg-PC bilayers may be ordered before it was dried.

(3) There exists the ripple phase in egg-PC bilayers below its phase transition temperature.

What we'll do next is to observe the egg-PC bilayers before being dried and its complex with protein. This may reveal the nature of the biomaterials in a higher degree.

${ }^{1} \mathrm{G}$. Binnig and H. Rohrer, Trends in Physics, edited by J. Janta and J. Panatoflicek (European Physical Society, Petit-Lancy, Switzerland, 1983), p. 38.

${ }^{2}$ S. M. Lindsay and B. J. Barris, J. Vac. Sci. Technol. A 6, 544 (1988).

${ }^{3}$ G. Travaglini, H. Rohrer, M. Amrein, and H. Gross, Surf. Sci. 181, 380 (1987).

${ }^{4}$ D. Dahn, W. Watanabe, B. Blackford, and M. H. Jericho, J. Vac. Sci. Technol. A 6, 548 (1988).

${ }^{5}$ A. M. Baró, R. Miranda, J. Alamán, N. García, G. Binnig, H. Rohrer, Ch. Gerber, and J. L. Carrascosa, Nature (London) 315, 253 (1985).

${ }^{6}$ A. O. Stemmer, R. Reichelt, A. Engel, J. P. Rosenbusch, M. Ringger, H. R. Hidber, and H.-J. Güntherodt, Surf. Sci. 181, 394 (1987).

${ }^{7}$ J. A. N. Zasadzinski, J. Schneir, J. Gurley, V. Elings, and P. K. Hansma, Science 239, 1013 (1988). 